\title{
Seletividade de Herbicidas Pré-Emergentes ao Pinhão-Manso (Jatropha curcas) $^{1}$
}

\author{
Selectivity of Pre-Emergence Herbicides to Physic Nut (Jatropha curcas)
}

ROCHA, P.R.R. ${ }^{2}$, SILVA, A.F. ${ }^{3}$, FARIA, A.T..$^{4}$, GALON, L. ${ }^{5}$, FERREIRA, E.A. ${ }^{6}$, FELIPE, R.S. ${ }^{7}$, SILVA, A.A. ${ }^{8}$ e DIAS, L.A.S. ${ }^{9}$

\begin{abstract}
RESUMO - Nos últimos anos, a área cultivada com pinhão-manso tem se expandido no Brasil, tendo em vista a sua utilização na produção de biocombustiveis. Um dos problemas enfrentados pelos produtores é a escassez de informações sobre a tolerância do pinhão-manso aos herbicidas registrados no Brasil. Diante disso, objetivou-se avaliar a tolerância de genótipos de pinhão-manso a herbicidas aplicados em pré-emergência da cultura. O experimento foi instalado em blocos casualizados, com quatro repetições, em esquema fatorial $3 \times 6$. O fator A foi composto pelos genótipos de pinhão-manso (Filomena, Gonçalo e Paraguaçu), e o fator B, pelos herbicidas (isoxaflutole, oxyfluorfen, sulfentrazone, pendimethalin e tebuthiuron) mais uma testemunha sem aplicação de herbicida. A intoxicação dos genótipos pelos herbicidas foi avaliada aos 19, 32, 40, 48, 56 e 64 dias após a aplicação dos tratamentos (DAT). Aos 64 DAT, avaliaram-se a altura, a área foliar e a massa seca da parte aérea das plantas. Constatou-se que os genótipos de pinhão-manso apresentaram niveis variáveis de tolerância aos herbicidas estudados. Isoxaflutole e tebuthiuron causaram severas injúrias aos genótipos avaliados e influenciaram negativamente todas as variáveis estudadas. Oxyfluorfen e pendimethalin mostraram-se mais seletivos, devendo ser avaliados em novos estudos a fim de serem recomendados para a cultura.
\end{abstract}

Palavras-chave: biocombustível, controle químico, fitointoxicação, tolerância

\begin{abstract}
In recent years, the areas cultivated with physic nut (Jatropha curcas) have expanded in Brazil, aiming at its use in biofuel production. However, one of the problems faced by the producers is the lack of studies on its tolerance to the herbicides registered in Brazil. The objective of this work was to evaluate the tolerance of physic nut genotypes to herbicides applied at pre-emergence. The experiment was arranged in a randomized block design, with four replications, in a factorial design, $3 \times$ 6. Factor A was composed by the genotypes (Filomena, Gonçalo and Paraguaçu), and factor $B$ by the herbicides (isoxaflutole, oxyfluorfen, sulfentrazone, pendimethalin and tebuthiuron), plus one non-treated control. Intoxication by the herbicides was evaluated at 19, 32, 40, 48, 56 and 64 days after treatments (DAT). Height, leaf area, and plant dry matter were evaluated at 64 DAT. It was verified that the jatropha genotypes have different tolerance to the herbicides studied. Isoxaflutole and tebuthiuron caused severe injuries to all genotypes and negatively affected all the variables evaluated. On the other hand, oxyfluorfen and pendimethalin proved to be selective to the crop and should be evaluated in further studies to be recommended to the crop.
\end{abstract}

Keywords: biofuel, chemical control, plant toxicity, tolerance.

1 Recebido para publicação em 20.12.2009 e na forma revisada em 12.11.2010.

2 Engo-Agro-., M.Sc., Doutorando no Programa de Pós-Graduação em Fitotecnia, Dep. de Fitotecnia, Universidade Federal de Viçosa - DFT/UFV, 36570-000 Viçosa-MG, <paulo.rocha@ufv.br>; ${ }^{3}$ Eng - -Agr ${ }^{0}$., D. Sc. em Fitotecnia; ${ }^{4}$ Estudante de Graduação do Curso de Agronomia, UFV; ${ }^{5}$ Engo-Agr ${ }^{\circ}$., D. Sc. Professor Adjunto, Universidade Federal do Pampa - UNIPAMPA, Itaqui-RS; ${ }^{6}$ Engo-Agr ${ }^{\circ}$., D.Sc. Bolsista PNPD, Universidade Federal dos Vales do Jequitinhonha e Mucuri - UFVJM, Diamantina-MG; ${ }^{7}$ Estudante de Graduação do Curso de Agronomia, UFV; ${ }^{8}$ Eng $^{-}-$Agr $^{\circ}$., D. Sc. Professor Associado, DFT/UFV; ${ }^{9}$ Engoํo-Agr ${ }^{\circ}$., D. Sc. Professor Adjunto, DFT/UFV. 


\section{INTRODUÇÃO}

A substituição parcial ou total do óleo diesel por biocombustiveis tornou-se questão de interesse estratégico, para suprir a escassez de combustiveis derivados de petróleo e reduzir os niveis de emissão de poluentes gasosos. A autorização do uso de misturas de biodiesel pelo governo brasileiro é o passo inicial para reduzir o uso de óleo diesel e desencadear ações de pesquisa sobre óleos vegetais (Corrêa et al., 2008).

Dentre as espécies potencialmente utilizáveis para essa finalidade, destaca-se como alternativa o pinhão-manso (Jatropha curcas), tendo em vista sua rusticidade e por apresentar longo ciclo reprodutivo, que pode chegar a 40 anos, com produtividade média de $5 \mathrm{t} \mathrm{ha}^{-1}$ (Teixeira, 2005; Arruda et al., 2004). O pinhãomanso pertence à família Euphorbiaceae; originário da America Tropical, encontra-se amplamente distribuído, tanto em áreas tropicais como subtropicais (Openshaw, 2000).

O cultivo de pinhão-manso vem crescendo nos últimos anos, e ainda há carência de informações sobre seu manejo visando obter alta produtividade com o mínimo de risco econômico e ambiental. Dentre os fatores abióticos e bióticos que interferem na produtividade do pinhão-manso, destaca-se a interferência das plantas daninhas. De modo semelhante a outras culturas perenes, as plantas jovens do pinhão-manso podem sofrer interferência negativa da comunidade infestante e ter o seu crescimento e desenvolvimento vegetativo comprometido, bem como a produção de sementes e de óleo (Erasmo et al., 2009). Na maioria das culturas de interesse econômico, a aplicação de herbicidas é o método mais utilizado para controle de plantas daninhas. Isso ocorre porque esse método é geralmente eficiente e rápido, tornando possivel o cultivo de grandes áreas, com pouca dependência de mão de obra. Todavia, a fim de utilizar o método químico de controle de plantas daninhas, são necessários estudos para selecionar herbicidas seletivos à cultura. A seletividade dos herbicidas às culturas é a base para o sucesso do controle químico das plantas daninhas, sendo considerada uma resposta diferencial de diversas espécies de plantas a determinado herbicida (Oliveira Jr., 2001; Das et al., 2003; Rizzardi et al., 2003).
A seletividade não pode ser determinada apenas pela verificação de sintomas visuais nas plantas. São conhecidos exemplos de herbicidas que podem reduzir a produtividade das culturas sem produzir-lhes efeitos visualmente detectáveis. Existem também casos de herbicidas que provocam injúrias bastante acentuadas às culturas, mas que permitem a plena recuperação das plantas e do potencial produtivo do cultivo (Silva et al., 2003). Dessa forma, quando se tem por objetivo estudar seletividade de herbicidas, é importante que se observem as intoxicações provocadas por eles, bem como os efeitos sobre o crescimento e a produtividade da planta cultivada (Negrisoli et al., 2004; Galon et al., 2009). Em pinhãomanso, poucos estudos dessa natureza foram realizados no Brasil, e ainda não existem herbicidas registrados para utilização nesta cultura; contudo, alguns herbicidas pré-emergentes são recomendados para outras culturas da mesma família botânica do pinhão-manso, como mamona, mandioca e seringueira (Agrofit, 2010). Em razão disso, espera-se que esses herbicidas apresentem seletividade ao pinhão-manso, como observado por Erasmo et al. (2009), em que os herbicidas diuron, trifluralin e isoxaflutole apresentaram potencial de utilização na cultura do pinhão-manso. Nesse sentido, este trabalho objetivou avaliar a sensibilidade de genótipos de pinhão-manso a alguns herbicidas aplicados em pré-emergência desta cultura.

\section{MATERIAL E MÉTODOS}

O experimento foi conduzido na casa de vegetação do Departamento de Fitotecnia da Universidade Federal de Viçosa, em Viçosa-MG, no delineamento experimental de blocos ao acaso com quatro repetições, em esquema fatorial $(3 \times 6)$. O fator A foi composto pelos genótipos de pinhão-manso (Filomena, Gonçalo e Paraguaçu) e o fator B pelos herbicidas isoxaflutole (113 $\left.\mathrm{g} \mathrm{ha}^{-1}\right)$, oxyfluorfen $\left(720 \mathrm{~g} \mathrm{ha}^{-1}\right)$, pendimethalin $\left(1.500 \mathrm{~g} \mathrm{ha}^{-1}\right)$, sulfentrazone (780 $\left.\mathrm{g} \mathrm{ha}^{-1}\right)$ e tebuthiuron ( $\left.1.100 \mathrm{~g} \mathrm{ha}^{-1}\right)$, além de uma testemunha sem aplicação de herbicida.

As unidades experimentais foram constituídas por vasos plásticos contendo $12 \mathrm{dm}^{3} \mathrm{de}$ solo, do tipo Latossolo Vermelho-Amarelo, previamente corrigido e adubado, de acordo 
com suas, características físicas e químicas (Tabela 1). Dois dias após a semeadura do pinhão-manso, foram aplicados, em pré-emergência, os herbicidas isoxaflutole, oxyfluorfen, pendimethalin, sulfentrazone e tebuthiuron. Utilizou-se pulverizador de pressão constante, equipado com barra contendo uma ponta de pulverização da série TT 110.02, calibrado para aplicar o equivalente a $150 \mathrm{~L} \mathrm{ha}^{-1}$ de calda. Foram semeadas duas sementes por vaso, e aos 10 dias após a emergência ajustou-se a densidade para uma planta por vaso.

A intoxicação das plantas pelos herbicidas (fitotoxicidade) foi avaliada visualmente aos 19, 32, 40, 48, 56 e 64 dias após a aplicação dos tratamentos (DAT), de acordo com escala percentual de notas, na qual 0 correspondeu à ausência de intoxicação e 100 à morte das plantas (SBCPD, 1995). Aos 64 DAT, avaliaram-se também a altura, a área foliar (AF) e a massa da seca da parte área das plantas. Para determinação da área foliar e da massa da parte aérea, elas foram seccionadas no nível do solo e separadas em caule e folhas, as quais foram levadas ao laboratório para determinação da área foliar em determinador eletrônico. O material foi acondicionado em sacos de papel e levado à estufa a $70{ }^{\circ} \mathrm{C}$ até atingir massa constante, para obtenção da massa da parte aérea.

As informações referentes à altura de planta, área foliar e massa da matéria seca da parte aérea foram transformadas em porcentagem relativa à testemunha não tratada com herbicida. Os dados obtidos foram submetidos à análise de variância pelo teste $\mathrm{F}$ e, quando houve diferença significativa entre os tratamentos,comparados entre si pelo teste de Tukey (Machado et al., 2002). Para efeito da intoxicação dos herbicidas ao longo do tempo, foram testados modelos de regressão. $\mathrm{O}$ ajuste do modelo aos dados foi baseado no fenômeno biológico, na significância e no coeficiente de determinação. Em todos os testes adotou-se o nivel de $5 \%$ de probabilidade.

\section{RESULTADOS E DISCUSSÃO}

Os genótipos de pinhão-manso responderam de forma diferenciada aos herbicidas, e todos os produtos causaram intoxicação à cultura em proporções variadas (Figuras 1, 2, 3 e 4). Nas plantas tratadas com o oxyfluorfen, observou-se intoxicação elevada aos 19 DAT, na forma de necrose no caule e deformações nos ápices das folhas cotiledonares das plantas. Contudo, observou-se boa recuperação das plantas, e aos 56 DAT os sintomas de intoxicação já estavam abaixo de $20 \%$ para todos os genótipos (Figura 1), confirmando resultados obtidos por Gonçalves et al. (2009). Dos genótipos avaliados, Filomena foi o que apresentou maior tolerância ao oxyfluorfen aos 19 DAT, destacando-se em relação aos demais.

Quanto à tolerância ao sulfentrazone, foram observados resultados semelhantes aos de oxyfluorfen: alta intoxicação inicial, mas com posterior recuperação das plantas. $\mathrm{Pa}-$ ra esse herbicida, todavia, não se observaram diferenças entre os genótipos (Figura 2). Sulfentrazone e oxyfluorfen inibem a enzima protoporfirinogênio oxidase (PROTOX); quando aplicados em pré-emergência, agem sobre o hipocótilo, o epicótilo e os meristemas foliares das plantas em emergência, não apresentando ação evidente ou significativa sobre os tecidos radiculares (Rodrigues \& Almeida, 2005). Essa característica provavelmente explica a recuperação das plantas intoxicadas por esses herbicidas.

Todos os genótipos de pinhão-manso apresentaram alta sensibilidade ao herbicida isoxaflutole. Aos 19 dias após a aplicação a intoxicação foi superior a $60 \%$ e acentuou-se ainda mais ao longo do tempo (Figura 3). O isoxaflutole é considerado um pró-herbicida,

Tabela 1 - Características químicas e físicas do solo. Viçosa-MG, 2009

\begin{tabular}{|c|c|c|c|c|c|c|c|c|c|c|c|c|c|c|}
\hline $\mathrm{pH}$ & $\mathrm{MO}$ & $\mathrm{P}$ & $\mathrm{K}$ & $\mathrm{Al}^{3+}$ & $\mathrm{Ca}^{2+}$ & $\mathrm{Mg}^{2+}$ & $\mathrm{H}+\mathrm{Al}$ & $\mathrm{SB}$ & $\begin{array}{c}\mathrm{CTC} \\
(\mathrm{t})\end{array}$ & $\begin{array}{c}\mathrm{CTC} \\
(\mathrm{T})\end{array}$ & $\mathrm{V}$ & Areia & Silte & Argila \\
\hline$\left(\mathrm{H}_{2} \mathrm{O}\right)$ & $\left(\mathrm{dag} \mathrm{kg}^{-1}\right)$ & $\left(\mathrm{mg} \mathrm{dm}^{-3}\right)$ & \multicolumn{8}{|c|}{$\left(\mathrm{cmol}_{\mathrm{c}} \mathrm{dm}^{-3}\right)$} & & \multicolumn{5}{c|}{$(\%)$} \\
\hline 4,7 & 2,10 & 0,9 & 25 & 1,0 & 0,5 & 0,2 & 5,12 & 0,76 & 1,76 & 5,88 & 13 & 62 & 10 & 38 \\
\hline
\end{tabular}




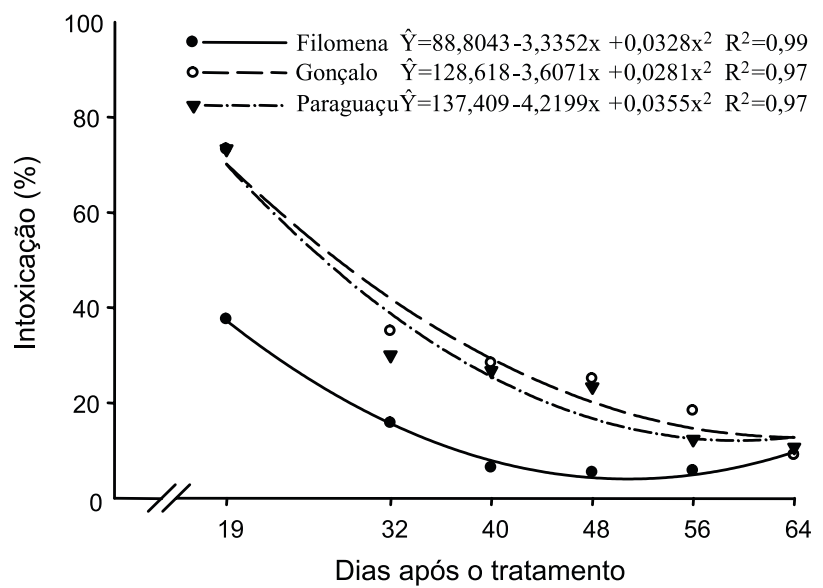

Figura 1 - Intoxicação de genótipos de pinhão-manso pelo herbicida oxyfluorfen. Viçosa-MG, 2009.

uma vez que é rapidamente convertido a diquetonitrila - molécula biologicamente ativa no controle de plantas daninhas (Marchiori Jr. et al, 2005). Este herbicida ocasiona a disfunção da sintese de pigmentos, levando a sintomas característicos de branqueamento de tecidos após a aplicação nas espécies suscetíveis, seguido de paralisação de crescimento e necrose (Pallet et al., 1998). É absorvido preferencialmente pelas raízes, embora o seja também pelas sementes (Cavalieri et al., 2008). Dos genótipos avaliados, Filomena foi o que apresentou menor intoxicação ao isoxaflutole, quando comparado aos demais. Gonçalo e Paraguaçu, aos 64 DAT, mostraram intoxicação superior a $80 \%$, ocorrendo a morte de algumas plantas desses genótipos. Os principais sintomas apresentados pelas plantas foram branqueamento, clorose generalizada e, posteriormente, necrose dos tecidos foliares.

O herbicida pendimethalin causou a menor intoxicação às plantas de pinhão-manso, com fitotoxicidade abaixo de 10\% durante todo o período de avaliação. Esse herbicida atua em tecidos meristemáticos, onde inibe o crescimento das células, a divisão celular, o crescimento da radícula e a formação das raízes secundárias nas plantas suscetíveis (Silva et al., 2007). A intoxicação média por pendimethalin foi de 5,7, 2,9 e 7,3\% para os genótipos Filomena, Gonçalo e Paraguaçu, respectivamente.

Dos herbicidas avaliados neste trabalho, tebuthiuron proporcionou a maior intoxicação

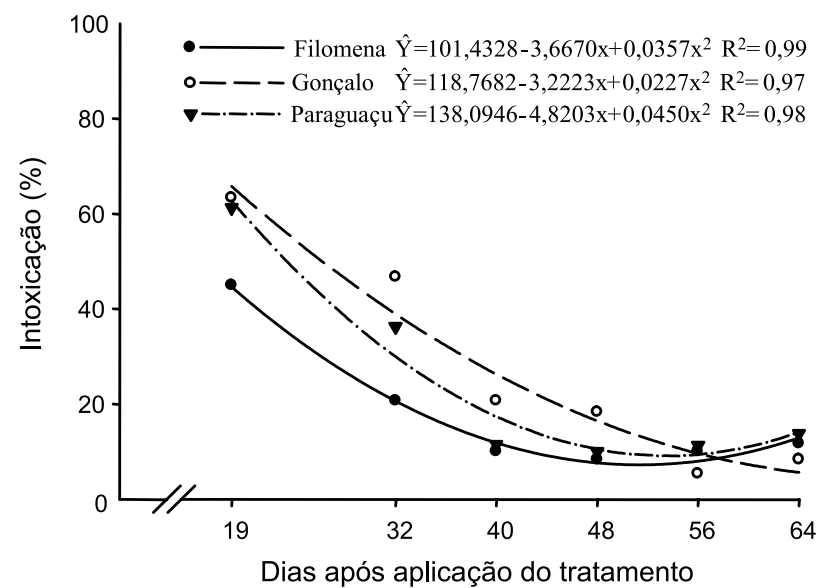

Figura 2 - Intoxicação de genótipos de pinhão-manso pelo herbicida sulfentrazone. Viçosa-MG, 2009

ao pinhão-manso, causando a morte da maioria das plantas tratadas. Os sintomas de intoxicação por esse produto foram, inicialmente, maiores no genótipo Gonçalo; aos 32 DAT, todos os genótipos avaliados apresentavam elevados indices de intoxicação, que se caracterizavam por clorose generalizada e redução severa no crescimento das plantas (Figura 4).

Tebuthiuron, isoxaflutole e sulfentrazone alteraram o crescimento de todos os genótipos. Pendimethalin não afetou os genótipos Filomena e Gonçalo, e oxyfluorfen reduziu o porte do genótipo Gonçalo. A redução da altura das plantas por isoxaflutole e por tebuthiuron foi superior a $80 \%$, enquanto o sulfentrazone mostrou comportamento intermediário para essa variável se comparado aos demais tratamentos (Tabela 2). A altura média das plantas na testemunha não tratada foi de 54, 44 e $45 \mathrm{~cm}$, para os genótipos Filomena, Gonçalo e Paraguaçu, respectivamente.

Efeitos de herbicidas inibindo o crescimento em altura de plantas de pinhão-manso foram também observados por Erasmo et al. (2009). Esses autores constataram que os herbicidas atrazine, oxyfluorfen e atrazine + S-metolachlor aplicados em pré-emergência reduziram a altura das plantas aos 33 DAT; aos 53 DAT, contudo, houve recuperação da altura das plantas tratadas com o oxyfluorfen.

A matéria seca da parte aérea das plantas (MSPA) na testemunha não tratada foi de 25,2, 24,6 e 25,4 g, para os genótipos Filomena, 


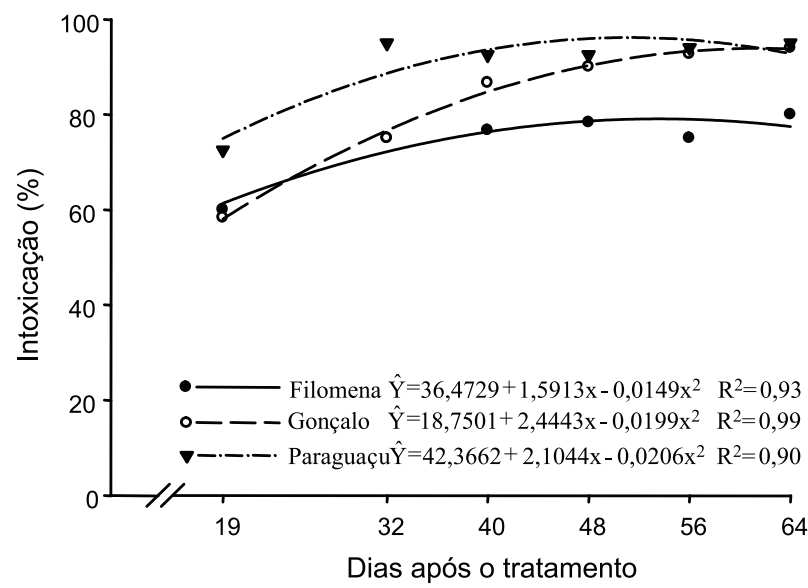

Figura 3 - Intoxicação de genótipos de pinhão-manso pelo herbicida isoxaflutole. Viçosa-MG, 2009.

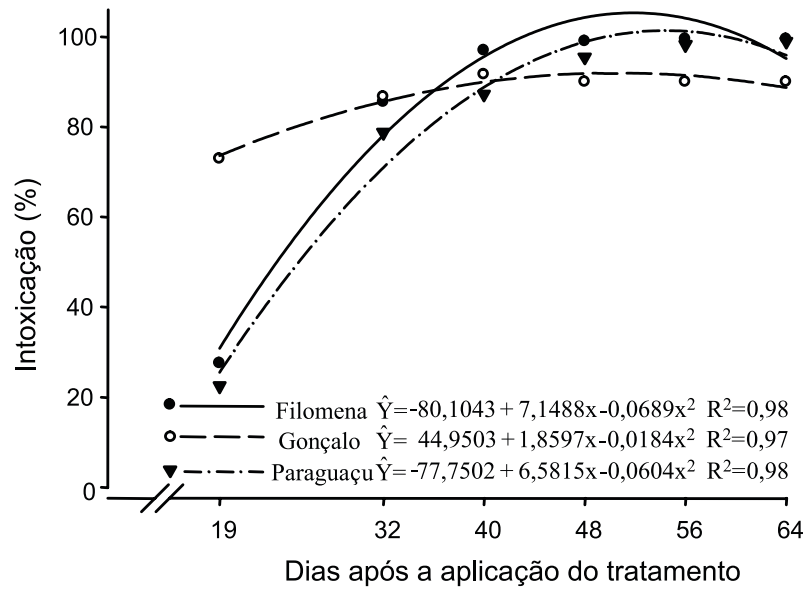

Figura 4 - Intoxicação de genótipos de pinhão-manso pelo herbicida tebuthiuron. Viçosa-MG, 2009.

Tabela 2 - Altura das plantas de pinhão-manso, em porcentagem em relação à testemunha não tratada, aos 64 dias após a aplicação de herbicidas pré-emergentes. Viçosa-MG, 2009

\begin{tabular}{|c|c|c|c|}
\hline \multirow{3}{*}{ Tratamento } & \multicolumn{3}{|c|}{ Genótipo } \\
\hline & Filomena & Gonçalo & Paraguaçu \\
\hline & \multicolumn{3}{|c|}{$\begin{array}{c}\text { Altura de plantas em relação à } \\
\text { testemunha }(\%)\end{array}$} \\
\hline Testemunha não tratada & $100,0 \mathrm{Aa}$ & $100,0 \mathrm{Aa}$ & $100,0 \mathrm{Aa}$ \\
\hline Oxyfluorfen & $64,3 \mathrm{Ba}$ & $79,4 \mathrm{ABa}$ & $67,7 \mathrm{Ba}$ \\
\hline Pendimethalin & $77,8 \mathrm{ABb}$ & $99,0 \mathrm{Aa}$ & $74,6 \mathrm{Bb}$ \\
\hline Sulfentrazone & $58,2 \mathrm{Ba}$ & $66,1 \mathrm{Ba}$ & $70,5 \mathrm{Ba}$ \\
\hline Isoxaflutole & $18,7 \mathrm{Ca}$ & $17,8 \mathrm{Ca}$ & $11,1 \mathrm{Ca}$ \\
\hline Tebuthiuron & $13,5 \mathrm{Ca}$ & $6,6 \mathrm{Ca}$ & $21,8 \mathrm{Ca}$ \\
\hline CV (\%) & \multicolumn{3}{|c|}{18,84} \\
\hline
\end{tabular}

Médias seguidas da mesma letra, maiúscula na coluna e minúscula na linha, não diferem pelo teste de Tukey a $5 \%$ de probabilidade.
Gonçalo e Paraguaçu, respectivamente. A aplicação dos herbicidas reduziu a MSPA, exceto para o genótipo Gonçalo no tratamento com pendimethalin. Assim como para altura de plantas, os herbicidas isoxaflutole e tebuthiuron foram os que influenciaram negativamente a MSPA, reduzindo esta variável em mais de 95\%, quando comparados à testemunha não tratada. Os genótipos responderam de maneira semelhante aos herbicidas isoxaflutole, sulfentrazone e tebuthiuron, porém apresentaram comportamento diferenciado com relação aos outros herbicidas. Gonçalo e Filomena tiveram menor redução na MSPA quando tratados com pendimethalin e oxyfluorfen, respectivamente (Tabela 3).

Todos os herbicidas promoveram redução da área foliar (AF) nos genótipos avaliados, quando comparados à testemunha não tratada. Tebuthiuron e isoxaflutole foram os que ocasionaram mais danos à AF, seguidos por sulfentrazone, oxyfluorfen e pendimethalin. Os genótipos responderam de maneira semelhante a tebuthiuron, isoxaflutole e sulfentrazone, porém Gonçalo e Paraguaçu foram os mais sensiveis ao oxyfluorfen e pendimethalin, respectivamente (Tabela 4). A AF da testemunha não tratada foi de $2.325,1.813$ e $1.933 \mathrm{~cm}^{2}$, para os genótipos Filomena, Gonçalo e Paraguaçu, respectivamente.

Os resultados deste trabalho permitem concluir que os genótipos de pinhão-manso apresentam níveis variáveis de tolerância

Tabela 3 - Massa seca da parte aérea (MSPA) de pinhão-manso, em porcentagem em relação à testemunha não tratada, aos 64 dias após a aplicação de herbicidas pré-emergentes. Viçosa-MG, 2009

\begin{tabular}{|l|c|c|c|}
\hline \multirow{2}{*}{\multicolumn{1}{|c|}{ Tratamento }} & \multicolumn{3}{|c|}{ Genótipo } \\
\cline { 2 - 4 } & Filomena & Gonçalo & Paraguaçu \\
\cline { 2 - 4 } & \multicolumn{3}{|c|}{ MSPA (\%) } \\
\hline Testemunha não tratada & $100,0 \mathrm{Aa}$ & $100,0 \mathrm{Aa}$ & $100,0 \mathrm{Aa}$ \\
\hline Oxyfluorfen & $74,4 \mathrm{Ba}$ & $64,6 \mathrm{Bab}$ & $52,6 \mathrm{Bb}$ \\
\hline Pendimethalin & $67,8 \mathrm{Bb}$ & $88,5 \mathrm{ABa}$ & $54,7 \mathrm{Bb}$ \\
\hline Sulfentrazone & $52,6 \mathrm{Ba}$ & $39,3 \mathrm{Ca}$ & $51,4 \mathrm{Ba}$ \\
\hline Isoxaflutole & $3,5 \mathrm{Ca}$ & $1,3 \mathrm{Da}$ & $0,5 \mathrm{Ca}$ \\
\hline Tebuthiuron & $0,6 \mathrm{Ca}$ & $2,9 \mathrm{Da}$ & $0,4 \mathrm{Ca}$ \\
\hline \multicolumn{3}{|c|}{$\mathrm{CV}(\%)$} & 25,03 \\
\hline
\end{tabular}

Médias seguidas da mesma letra, maiúscula na coluna e minúscula na linha, não diferem pelo teste de Tukey a $5 \%$ de probabilidade. 
Tabela 4 - Área foliar das plantas de pinhão-manso, em porcentagem em relação à testemunha não tratada, aos 64 dias após a aplicação de herbicidas pré-emergentes. Viçosa-MG, 2009

\begin{tabular}{|c|c|c|c|}
\hline \multirow{3}{*}{ Tratamento } & \multicolumn{3}{|c|}{ Genótipo } \\
\hline & Filomena & Gonçalo & Paraguaçu \\
\hline & \multicolumn{3}{|c|}{ Área foliar (\%) } \\
\hline Testemunha não tratada & $100,0 \mathrm{Aa}$ & $100,0 \mathrm{Aa}$ & $100,0 \mathrm{Aa}$ \\
\hline Oxyfluorfen & $76,8 \mathrm{~B} \mathrm{a}$ & $62,3 \mathrm{BCb}$ & $75,6 \mathrm{Ba}$ \\
\hline Pendimethalin & $73,9 \mathrm{Ba}$ & $75,4 \mathrm{Ba}$ & $59,9 \mathrm{Cb}$ \\
\hline Sulfentrazone & $50,5 \mathrm{Ca}$ & $49,9 \mathrm{Ca}$ & $50,1 \mathrm{Ca}$ \\
\hline Isoxaflutole & $6,8 \mathrm{Da}$ & $3,0 \mathrm{Da}$ & $2,0 \mathrm{Da}$ \\
\hline Tebuthiuron & $0,4 \mathrm{Da}$ & $4,6 \mathrm{Da}$ & $0,4 \mathrm{Da}$ \\
\hline $\mathrm{CV}(\%)$ & \multicolumn{3}{|c|}{13,60} \\
\hline
\end{tabular}

Médias seguidas da mesma letra, maiúscula na coluna e minúscula na linha, não diferem pelo teste de Tukey a $5 \%$ de probabilidade.

aos herbicidas estudados. Isoxaflutole e tebuthiuron causaram injúrias severas às plantas, influenciando negativamente todas as variáveis avaliadas; já oxyfluorfen e pendimethalin mostraram potencial para utilização em áreas cultivadas com pinhãomanso e devem ser avaliados em novos estudos nessa cultura.

\section{LITERATURA CITADA}

AGROFIT. Sistema de Agrotóxicos Fitossanitários. 2010. Disponível em: <http://extranet.agricultura.gov.br/agrofit > Acesso em: 12 de abril de 2010.

ARRUDA, F. P. et al. Cultivo de pinhão manso (Jatropha curcas L.) como alternativa para o semi-árido nordestino. R. Bras. Oleag. Fibrosas, v. 8, n. 1, p. 789-799, 2004.

CAVALIERI, S. D. et al. Tolerância de milhos híbridos de milho ao herbicida isoxaflutole. Planta Daninha, v. 26, n. 4, p. 901-909, 2008.

CORRÊA, I. M. et al. Desempenho de motor diesel com misturas de biodiesel de óleo de girassol. Ci. Agrotecnol., v. 32, n. 3 , p. $923-928,2008$.

DAS, A. C. et al. Effect of the herbicides oxadiazon and oxyfluorfen on phosphates solubilizing microorganisms and their persistence in rice fields. Chemosphere, v. 53, n. 5 , p. 217-221, 2003.

ERASMO, E. A. L. et al. Tolerância inicial de plantas de pinhão-manso a herbicidas aplicados em pré e pósemergência. Planta Daninha, v. 27, n. 3, p. 571-580, 2009.
GALON, L. et. al. Seletividade de herbicidas a genótipos de cana-de-açúcar. Planta Daninha, v. 27, p. 1083-1093, 2009. (Edição Especial).

GONÇALVES, K. S. et al. Seletividade do oxyfluorfen para a cultura do pinhão-manso. Planta Daninha, v. 27, p. 1111-1116, 2009. (Edição Especial)

MACHADO, A. A. et al. Sistema de análises estatísticas para Windows - WINSTAT (Versão 2.11). Pelotas:

Universidade Federal de Pelotas, 2002.

MARCHIORI JR., O. et al. Efeito residual de isoxaflutole após diferentes períodos de seca. Planta Daninha, v. 23, n. 3, p. 491-499, 2005.

NEGRISOLI, E. et al. Seletividade de herbicidas aplicados em pré-emergência na cultura de cana-de-açúcar tratada com nematicidas. Planta Daninha, v. 22, n. 4, p. 567-575, 2004.

PALLET, K. E. et al. The mode of action of isoxaflutole. I. Physiological effects, metabolism, and selectivity. Pestic. Biochem. Physiol., v. 62, n. 2, p. 113-124, 1998.

OLIVEIRA JR., R.S. Seletividade de herbicidas para culturas e plantas daninhas. In: OLIVEIRA JR, R. S.; CONSTANTIN, J. (Orgs.). Plantas daninhas e seu manejo. Guaíba: Agropecuária, 2001. p. 291-314

OPENSHAW, K. A review of Jatropha curca: an oil plant of unfulfilled promise. Biomassa Bioenergy, v. 19, n. 1, p. $1-15,2000$.

SILVA, A. A. et al. Controle de plantas daninhas. In: ASSOCIAÇÃO BRASILEIRA DE ENSINO SUPERIOR (ABEAS). Curso de proteção de plantas. Brasília: 2003. $260 \mathrm{p}$

SILVA, A. A.; FERREIRA, F. A.; FERREIRA, L. R Herbicidas: classificação e mecanismos de ação. In: SILVA, A. A.; SILVA, J. F. Tópicos em manejo integrado de plantas daninhas. Viçosa, MG: Universidade Federal de Viçosa, 2007. p. 58-117.

RIZZARDI, M. A. et al. Ação de herbicidas sobre mecanismos de defesa das plantas aos patógenos. Ci. Rural, v. 33, n. 5 , p. $957-965,2003$.

RODRIGUES, B. N.; ALMEIDA, F. S. Guia de herbicidas. 3.ed. Londrina: IAPAR, 2005. 591 p.

SOCIEDADE BRASILEIRA DA CIÊNCIA DAS PLANTAS DANINHAS - SBCPD. Procedimentos para instalação, avaliação e análise de experimentos com herbicidas. Londrina: $1995.42 \mathrm{p}$.

TEIXEIRA, L. C. Potencialidades de oleaginosas para produção de biodiesel. Inf. Agropec., v. 26, n. 229, p. 18-27, 2005.

VARGAS, L.; FLECK, N. G. Seletividade de herbicidas do grupo químico dos ariloxifenoxipropionatos a cereais de inverno. Planta Daninha, v. 17, n. 1, p. 41-51, 1999. 